\title{
Understanding Hysteresis in Human UDP-Glucose Dehydrogenase
}

\author{
Zachary A. Wood, Nathaniel R. Beattie, Phillip M. Gross, Renu Kadirvelraj, \\ Nicholas D. Keul, Weston E. McDonald \\ Department of Biochemistry \& Molecular Biology, University of Georgia, Athens, GA 30602, USA
}

Human UDP-glucose dehydrogenase (hUGDH) catalyzes the $\mathrm{NAD}^{+}$-dependent production of UDP-glucuronic acid, an essential substrate in drug metabolism. Some cancers have been shown to co-opt glucuronidation as an effective drug resistance mechanism. Thus, controlling hUGDH activity is a promising strategy for sensitizing some drug resistant tumors to existing chemotherapeutics. The progress curves of the hUGDH reaction reveal a significant lag in enzyme activity. Such hysteresis occurs when an enzyme undergoes a slow transition from an inactive $\left(\mathrm{E}^{*}\right)$ to an active $(\mathrm{E})$ conformation when the reaction is initiated. Understanding the nature of the $\mathrm{E}^{*}$ state may illuminate new strategies for regulating hUGDH. The $\mathrm{E}^{*}$ state in hysteretic enzymes can be caused by: i) the substrate-induced association of inactive subunits to form an active complex; ii) the dissociation of an inactive aggregate; or iii) the substrate-induced isomerization of an enzyme from an inactive state. Here we use transient state analysis, sedimentation velocity and a new crystal structure to characterize the $\mathrm{E}^{*}$ state of hUGDH. Combined with steady state analysis and binding studies, we show that the hysteretic transition results produces an asymmetric complex that displays negative cooperativity. This work identifies the $\mathrm{E}^{*}$ state as a possible target to controlling the activity of hUGDH. For more information, see the accompanying poster presented by $\mathrm{Mr}$. Nathaniel R. Beattie that tests this model (abstract no.171). 